\title{
Consequencs of a Dependence of GRB Properties on Local Metallicity
}

\author{
Enrico Ramirez-Ruiz*, Andrew W. Blain ${ }^{\dagger}$ and Davide Lazzati* \\ ${ }^{*}$ Institute of Astronomy, Madingley Road, Cambridge CB3 OHA, England \\ ${ }^{\dagger}$ Department of Astronomy, California Institute of Technology, Pasadena, CA 91125, USA
}

\begin{abstract}
We report a correlation between the isotropic equivalent energy of GRBs and their position offset from their host galaxies. This is possibly due to a dependence of the end point of massive stellar evolution on metallicity. If confirmed in further host observations, this correlation will both complicate interpretation of GRBs as tracers of cosmic star formation, and potentially allow a new probe of the astrophysics in high-redshift galaxies.
\end{abstract}

\section{THE BIRTH PLACE OF GAMMA-RAY BURSTS}

Evidence is accumulating that GRBs are intimately linked with the deaths of massive stars. For the longburst afterglows localized so far, the host galaxies show signs of the ongoing star formation activity necessary for the presence of young, massive progenitor stars $[1,2,3]$. The physical properties of the afterglows, their locations in host galaxies [4], iron line features, and evidence for supernova components several weeks after the burst $[5,6]$. strongly support the idea that the most common GRBs are linked to the collapse of massive stars.

The circumburst medium provides a natural laboratory for studying GRBs. Stars that readily shed their envelopes have short jet-crossing times and are more likely to produce a GRB. Stars with less radiative mass loss retain a hydrogen envelope, in which a poorly collimated jet is likely to lose energy and fail to breaking out of the star [7]. Finding useful diagnostics for the progenitors is simplified if the metallicity of and physical conditions in the local ISM influences the evolution of the progenitor. GRBs occur close to the birth sites of their short-lived progenitors, and so their evolution is likely to be affected only by local properties of the host galaxy [8].

Here, we show that bursts located closer to the center of their parent galaxies have smaller isotropic equivalent energies (or broader jets), and so progenitors in inner and outer galactic locations may be intrinsically different (see Figure 1). We suggest that this could be the outcome of abundance gradients in the host galaxy.

\section{ELEMENT ABUNDANCES FROM THE LOCAL UNIVERSE TO HIGH REDSHIFTS}

An exciting recent development in observational cosmology has been the extension of studies of abundances from the local Universe to high redshifts. The dependence of metallicity on environment appears to be stronger than on the redshift of formation: galaxies selected using the same techniques have metallicities rather independent of redshift, and old stars are not necessarily metal-poor [9]. Chemical abundances within different galaxies depend strongly on luminosity and environment [9]. From the center to the outermost $10 \mathrm{kpc}$, metallicity typically decreases by a factor of ten. A comparable change in metallicity only occurs over a range of a factor of a thousand in luminosity (see Fig. 5 of [9]). This is a much greater range of luminosity than displayed by moderate-redshift GRB host galaxies, which usually have magnitudes $R \approx$ 25 . These host galaxies are UV-bright [10], and so may exhibit comparable abundance gradients to their local counterparts. Drawing inferences about GRB hosts from local galaxies is difficult, however, since both merging and secular evolution are likely to be important and will complicate a direct comparison. Nonetheless, a direct association between abundance gradients in GRB hosts and in local galaxies could be responsible for the correlation presented in Figure 1.

Low-metallicity stars, which are likely to be more prominent in the outskirts of the galaxy, are smaller and have less mass loss than their metal-rich counterparts. Both properties inhibit the loss of angular momentum [7], and so low-metallicity stars are likely to be rotating rapidly. Equatorial accretion may thus be delayed and 


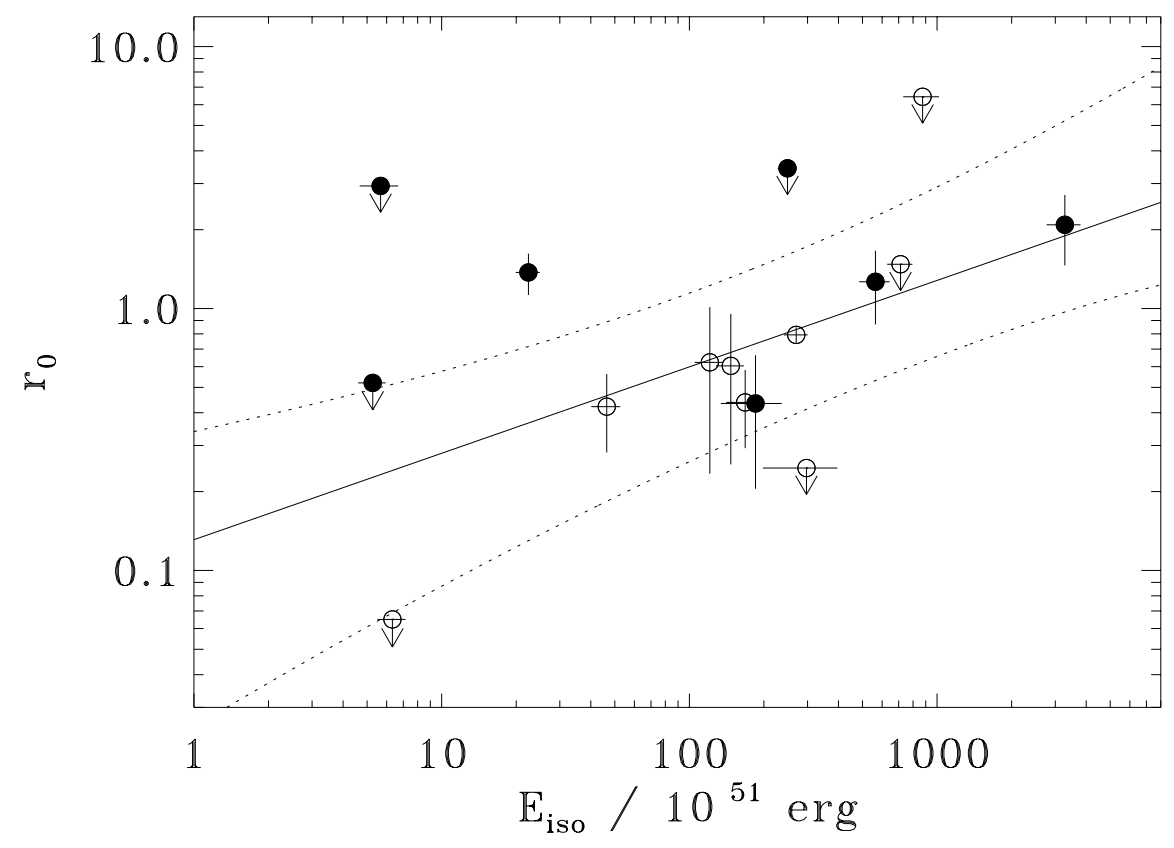

FIGURE 1. The projected observed offset of GRBs from their parental galaxy as a function of the burst isotropic equivalent energy. The center of the assigned host is determined as the centroid of the brightest component of the host system. The fractional isophotal offsets $r_{0}$ are the observed offsets $R_{0}$ normalized by the host half-light radius. Solid and dotted lines mark the center and $1 \sigma$ widths of the best-fit model distributions parameters. The filled circles are bursts that occur in the most irregular, possibly merging galaxies, while the empty circles are bursts with more regular hosts. There is a tentative trend: the inner most bursts seem to be less energetic.

a funnel may be produced along the rotation axis. For higher rotational velocities this evacuated region will be more collimated, reducing the jet opening angle. Furthermore, for a given mass-loss rate, the lower the metallicity, the higher both the WR stellar mass, and the mass threshold for the removal of the hydrogen envelope by stellar winds. These effects all increase the mass of the helium core and favor black hole formation [7, 11]. If there are abundance gradients in the hosts, then the likely metallicity dependence of both black-hole formation and rotation suggests that GRBs in outer galactic locations may be more energetic (greater helium core mass) or less collimated (faster rotation) than those close to the galactic center. In the local Universe, regular galaxies are found to have steeper abundance gradients than those with complex morphologies [12]. Indeed, it is reassuring that the most regular GRB host galaxies (shown as open circles in Fig. 1) firmly support the trend between $E_{\text {iso }}$ and $r_{0}$. Note that the scatter in Fig. 1 can be due to the dependence of metallicity on luminosity. A more detailed analysis of the underlying reasons for the correlation requires a large and unbiased sample of GRBs hosts, and knowledge of both the underlying GRB and afterglow luminosity functions.

\section{THE EFFECTS OF METALLICITY}

What are the potential effects of a significant dependence of GRB luminosity, as detected by unextinguished $\gamma$ ray photons, on their location in the host galaxy, which could reflect the metallicity of their progenitors? The most significant is a potential offset between the true star-formation rate and that traced by GRB. If GRBs in outlying, low-metallicity environments and in low-mass galaxies are more luminous, then they are likely to be overrepresented in GRB samples, and especially in the bright BATSE catalog, as compared with those in highmetallicity environments.

The radial dependence of metallicity $Z$ in lowredshift spiral [12] and elliptical galaxies [13], is $Z \propto \exp (-1.9 R / \bar{R})$, while the dependence of metallicity at fixed radius on enclosed mass $M_{\text {enc }}$ in spiral galaxies derived from Fig. 4 of [13] is $Z \propto M_{\mathrm{enc}}^{\simeq-0.5}$. These 
functions both depend strongly on radius. Therefore, it is likely that local environmental effects will overcome global enrichment effects [9], but that there will be a gradual increase in the typical luminosity of GRBs with increasing redshift (see [14]).

Low-mass galaxies are likely to have statistically lower metallicities and thus contain more luminous GRBs than high-mass galaxies. As galaxy mass is expected to build up monotonically through mergers, then it is possible that the highest-redshift GRBs could be systematically more luminous due to the lower mass of their hosts, perhaps by a factor of $2-3$ at $z \simeq 3$. This effect is likely to be more significant than, but in the same direction as, the global increase in metallicity with cosmic time.

The most luminous GRBs of all could be associated with metal-free Population-III stars; however, their very high redshifts would make examples difficult to find even in the Swift catalog of hundreds of bursts.

Star-formation activity is likely to be enhanced in merging galaxies. In major mergers of gas-rich spiral galaxies, this enhancement takes place primarily in the inner kpc, as bar instabilities drive gas into the core [15]. Metallicity gradients in the gas are likely to be smoothed out, both by mixing prior to star formation, and by $\mathrm{SN}$ enrichment during the burst of activity. GRB luminosities could thus be suppressed in such well-mixed galaxies, making GRBs more difficult to detect in these most luminous objects, in which a significant fraction of all high-redshift star formation is likely to have occurred. Shocks in tidal tails associated with merging galaxies are also likely to precipitate the formation of high-mass stars, yet as tidal tails are likely to consist of relatively low-metallicity gas, it is perhaps these less intense sites of star-formation at large distances from galactic radii that are more likely to yield detectable GRBs.

For star formation taking place in both merging and quiescent high-redshift galaxies, there should thus be a bias in favor of detecting GRBs at a greater projected distance from the host galaxy than the mean radius of the star-formation activity. Hence, based on the correlation shown in Figure 1, we predict that the radial distribution of a large sample of GRBs around their host galaxies should be considerably more extended than the signatures of star-formation regions within the host, such as blue colors, location of $\mathrm{H} \alpha$ emission, intense radio emission etc. This might have the unfortunate consequence of making GRBs more difficult to use as clean markers of high-redshift star-formation activity. Detailed observations of the astrophysics of individual GRB host galaxies may be essential before a large sample of bursts can be interpreted. More optimistically, the astrophysics of star formation in high-redshift galaxies could perhaps be studied using the intrinsic properties of a well-selected population of GRB with deep, resolved host galaxy im- ages.

If confirmed in detailed studies, a metallicity selection effect for GRBs may be able to explain the differences between the star-formation rate inferred from observations of galaxies $[16,17]$, which tend not to increase with redshift beyond $z \simeq 2$, and the rate inferred from GRB counts assuming a variability-luminosity relation $[18,14]$, which continues to increase to the highest redshifts. This increase may reflect a bias to detecting high-redshift GRBs in more numerous, low-mass, lowmetallicity high-redshift galaxies.

Another test of the effect could be provided by a comparison of the luminosity function of GRB host galaxies with that of the total galaxy luminosity function over the same redshift range. If there is a bias towards the discovery of GRBs in low-metallicity regions, then the GRB host galaxy luminosity function should be biased to low luminosities by an increasing amount as redshift increases.

We thank G. Denicoló, M. Pettini, M. J. Rees, C. Tout, and N.Trentham for useful comments and suggestions. ERR acknowledges support from CONACYT, SEP and the ORS foundation. AWB thanks the Raymond \& Beverly Sackler Foundation for financial support.

\section{REFERENCES}

1. Kulkarni, S. R. et al., Nature, 395, 663 (1998).

2. Fruchter, A. S. et al., ApJ, 520, 54 (1999).

3. Berger, E., Kulkarni, S. R., Frail, D. A., ApJ, 560, 652 (2001).

4. Bloom, J. S., Kulkarni, S. R., Djorgovski, S. G., AJ, 123, 1111 (2002).

5. Bloom, J. S. et al., Nature, 401, 453 (1999)

6. Reichart, D. E., ApJ, 521, L111 (1999).

7. MacFadyen, A. I., Woosley, S. E., ApJ, 524, 262 (1999)

8. Ramirez-Ruiz, E., Lazzati, D., Blain, A. W., ApJ, 565, L9 (2002)

9. Pettini, M., Proc. of the 'The promise of First', Pilbratt, Cernicharo, Heras \& Prusti eds., ESA SP-460 (2001).

10. Trentham, N., Ramirez-Ruiz, E., Blain, A. W., MNRAS in press (astro-ph/0204350) (2002).

11. Ramirez-Ruiz, E., Dray, L., Madau, P., Tout, C. A., MNRAS, 327, 829 (2001).

12. Zaritsky, D., Kennicut, R. C., Huchra J. P., ApJ, 420, 87 (1994).

13. Henry, R. B. C., Worthey, G., PASP, 111, 919 (1999).

14. Lloyd-Ronning, N. M., Fryer, C. L., Ramirez-Ruiz, E., ApJ in press (astro-ph/0108200) (2002).

15. Mihos, J. C., Hernquist, L., ApJ, 431, L9 (1994).

16. Steidel, C. C., Adelberger, K. L., Giavalisco, M., Dickinson, M., Pettini, M., ApJ, 519, 1 (1999).

17. Blain, A. W., Smail, I., Ivison, R. J., Kneib, J.-P., 1999, MNRAS, 302, 632 (1999).

18. Fenimore, E. E., Ramirez-Ruiz, E., ApJ submitted (astro-ph/0004176) (2001). 